\title{
EFFECT OF GINGER AND GINSENG RHIZOMES ON LIVER FUNCTION IN HYPERLIPIDEMIC RATS
}

\author{
M.A. Hbib, A.M. Farid, H.El.A. Farid and S.H.M. Hebisha \\ Department of Biochemistry Faculty of Agric. Fac. Menoufia. Unv.
}

Received: Nov. 12, 2019

Accepted: Nov. 24, 2019

\begin{abstract}
The study aimed to investigate the effect of ginger and ginseng rhizome on liver functions in hyperlipidemic rats. The addition of $5 \%$ or $10 \%$ ginger and ginseng to the feed of hyperlipidemic rats produced decreased total cholesterol level, Triglycerides as well as HDL-C, LDL-C and VLDL-c levels.

The liver enzymes AST, ALT and ALP showed an increase in their activities in the rats feed on hyperlipidemic diet comparing with normal control. Using ginger and ginseng decreased the activities of these enzymes compared to the positive control. AST/ALT ratio recorded values under 5 (between 1.02 and 1.07) this proved that the harmful of induced hyperlipidemic effect liver. Hyperlipidemic diet produced a decreased in total protein and albumin in blood. The addition of ginger and ginseng increased the amount of total protein and albumin compared to positive control.
\end{abstract}

Key word: lipid, liver, kidney, antioxidant, hyperlipidemic, cholesterol, phenolic, enzyme, rhizome.

\section{INTRODUCTION}

Vitamin $\mathrm{C}$ and total carotenoids content were found to be $\mathbf{1 0 . 9 7}$ and $\mathbf{9 2 . 9 6}$ $\mathrm{mg} / 100 \mathrm{~g}$ in dry ginger rhizome respectively (Gopalan et al., 2004). Matsuda et al., (2009) and Islam and Choi, (2008) reported that zingiber officinale Roscoe (Zingiberaceae) is the most widely used spice worldwide. It has been used as an antioxidant, anti-diabetic and anti-hyperlipidemic. Ginger has gained interest for its potential to treat various aspects of cardiovascular disease, and the in vitro and animal data supporting the anti-inflammatory, antioxidant, antiplatelet, hypotensive, and hypolipidemic effects of this condiment have been reviewed by (Nicoll and Henein 2009) and (Bhandari et al., 1998).

Administering an aqueous extract of raw ginger (administered daily, 500 $\mathrm{mg} / \mathrm{kg}$ intraperitoneally) to streptozotocin-induced diabetic rats lowered their serum glucose, cholesterol, and triacylglycerol levels, urine, protein levels, water intake, and urine output and prevented the weight loss associated with diabetes in this model (Al-Amin et al., 2006) and (Fuhrman et al., 2000).

Total saponin, panaxadiol, and panaxatriol from ginseng have been able to protect cardiomyocytes from ischemia and reperfusion injuries (Zhang et al., 2009). Cardiac injury in diabetes induced by streptozotocin has been prevented by ginsenoside Rb1 (Kim et al., 2012).

Sang et al., (2015) reported that the Korean red ginseng manufactured by the traditional preparation method has its own chemical component characteristics. The ginsenoside content of the red ginseng is Rg1:3.3 mg/g, Re:2.0 mg/g, $\mathrm{Rb} 1: 5.8 \mathrm{mg} / \mathrm{g}, \mathrm{RC}: 1.7 \mathrm{mg} / \mathrm{g}, \mathrm{Rb2:2.3} \mathrm{mg} / \mathrm{g}$, and $\mathrm{Rd}: 0.4 \mathrm{mg} / \mathrm{g}$, respectively.

Nadeem et al., (2009) and Arif et al., (2012) observed that the amino sugars have an inhibitory effect on protein glycosylation in blood, attenuating various complications related to diabetes mellitus. and hyperglycemia. 
Lee et al., (2006), Luo and fang (2008) reported that ginseng polysaccharides have immunomodulation, anti - fatigue, antitumor, anti-adhesive, antioxidant, antiulcer, anti-radiation, hepatoprotective, hypoglycemic activities and anti-hyperlipidemic activities.

The present study is investigating the effect of supplement rat diet with Sheep tail fat or Coconut Oil on the liver function.

\section{MATERIAL AND METHODS}

The roots of ginger (Zingiber officinale) and Panax ginseng were purchased from local market.

Adult male albino rats (30) were acclimatized to laboratory conditions and divided into 6 groups each having 5 rats as follows:

- Group1: Rats kept without any treatments as control fed on standard diet, named normal control or negative control (I).- Group2: Rats feed hyperlipidemic diet, hyperlipidemia or positive control (II). - Group3: Rats fed hyperlipidemic diet plus ginger $5 \%$ powder in food (III). - Group4: Rats fed hyperlipidemic diet plus ginger $10 \%$ powder in food (IV). - Group5: Rats feed hyperlipidemic diet plus ginseng 5\% powder in food (V). - Group6: Rats fed hyperlipidemic diet plus ginseng $10 \%$ powder in food (VI).

After 15 and 30 days at end of the experimental blood samples were taken plasma and serum sample were separated and kept for analyses. The animals were sacrificed and the organs (liver, kidneys and heart) were weighted.

Total cholesterol (TC) and triglycerides (TG) were determined according to the method described by Fossati and Prencipe (1982).

HDL-c was determined according to Lopez, et al., (1977).
LDL-c and VLDL-c were calculated according to the formula of Fiedewaid, et al., (1972).

LDL-C $=$ Total cholesterol $-($ HDL-c + triglyceride / 5)

vLDL-c = triglyceride $/ 5$

\section{Determination of liver function.}

Determination of AST and ALT activity was conduct by the method described by Young, (1990). ALP activity was determined according to Moss, et al., (1987). Total protein was determined according to Schultze and Heremans, (1966). Determination of albumin (Alb) concentration was carried and according to Cannon, et al., (1974).

\section{Statistical analysis:}

Statistical differences were determined by one-way analysis of variance (ANOVA), followed by post-hoc comparisons (LSD test). Differences were considered statistically significant at $\mathbf{P}$ $<0.05$, (Landue and Everitt 2004).

\section{RESULTS AND DISCUSSION}

The obtained results Table (1) illustrated that TC, TG, HDL, LDL, and vLDL-cholesterol for normal control were $160,122,43,91.4$ and $24.4 \mathrm{mg} / \mathrm{dl}$ respectively in plasma after 30 days. Feeding on high fat diet and cholesterol diet (positive control) caused highly significant increase in TC, TG, HDL, LDL, and vLDLc compared with negative control. The results showed that total cholesterol level was significant decreased to 200, 183, 208 and $190 \mathrm{mg} / \mathrm{dl}$ in groups $5 \%$ ginger and ginseng respectively after 30 days. Compared with hypercholesterolemic group (positive control). Triglyceride also was significantly decreased to191.9, 172, 196, and $182 \mathrm{mg} / \mathrm{dl}$ for all treatment groups respectively, compared with the positive control (303 mg/dl). 


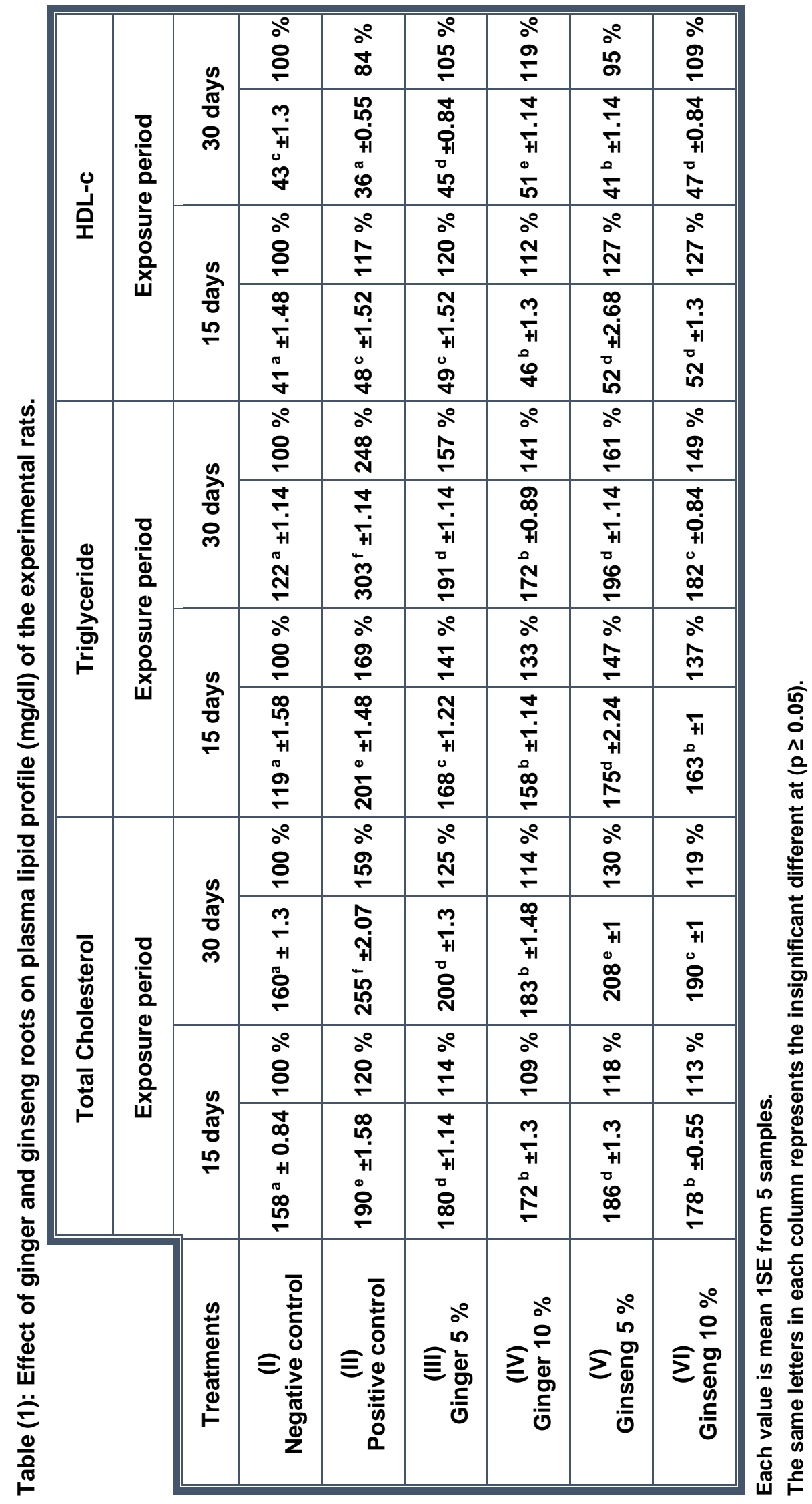


HDLC amounts increased of all treatments ginger $5 \& 10 \%$ and ginseng $5 \&$ $10 \%$ compared to positive control. The results showed that feeding the rats on food containing $10 \%$ ginger produced the maximum increased HDL-c $(51 \mathrm{mg} / \mathrm{dl})$ followed by the $10 \%$ ginseng $(47 \mathrm{mg} / \mathrm{dl}$ ) comparing to positive control $(36 \mathrm{mg} / \mathrm{dl})$ after 30 days.

Table (2) LDL-C and vLDL-c produced significant decreases in all treatments as compared to the positive control.

From the above it can be noticed that ginger and ginseng roots showed readjustment for TC, TG, LDL-C and vLDL-c levels relative to normal control. The levels of total cholesterol in blood especially lipoprotein LDL-c fraction are the most crucial health factor which are correlated with many health disorders. Liver expectancy may represent an integral response to its values (Niu et al., 2008).

\section{liver functions}

ALT as well as AST are used in the evaluation of hepatic disorders. The increase of the activities of AST and ALT in blood may be mainly due to the leakage of these enzymes from the liver cytosol into the blood stream.

Table (3) show that ginger and ginseng roots decreased the activities of AST, ALT with their ratio and alkaline phosphatase (ALP) on liver compared to hypercholesterolemic rats.

Data in Table (3) indicated that AST activity were 25 and $60 \mathrm{U} / \mathrm{L}$ for negative and positive controls respectively, after 15 days. It reached to 26 and $82 \mathrm{U} / \mathrm{L}$ for the two former controls after $\mathbf{3 0}$ days of experiment period. The addition of one of $5 \%$ and $10 \%$ ginger or ginseng roots to hyperlipidemic diet revealed a significant reduction in AST activity compared to positive control.
Increasing of ALT (Table 3) activity in hyperlipidemic rats compared to their negative control indicated that hyperlipidemia might be induced due to liver dysfunction. The activity of ALT in hypercholestrolemic rats was nearly treble that of normal control. The addition of $5 \%$ or $10 \%$ ginger and ginseng roots to hyperlipidemic diet revealed a significant reduction in ALT activity compared with the positive control. The stimulated activity of ALT indicates liver cells necrosis and the magnitude of increase correlated with the extent of necrosis. (EL-Abd et al., 2007).

Data in Table (3) show that AST I ALT ratio recorded values under 5 (between 1.02 and 1.07), this proved that the harmful of induced hyperlipidemic affect liver but not heart (ratio more than 20) as reported by Murray et al., (2006).

Alkaline phosphatase activity (ALP) showed stimulation in the activity of positive control relative to normal. From date presented in Table (3) it is interesting to notice that the tested plants, ginger and ginseng roots, produced significant increase in the activity of plasma ALP compared with the normal control after 15 and 30 days of treatment. While comparing the results with positive control an opposite trend was noticed. Ahmed et al., (2000) found that ginger $0.5 \%$ showed a significant decrease in alkaline phosphates activity in rats.

Plasma total protein, albumin, globulin level and A/G ratio.

Data in Table (4) showed a significant decrease for all groups in the total protein and albumin comparing with normal control.

This decrease in serum albumin (Alb) may be due to the effect of fat and cholesterol rich-diet on RNA and protein synthesis. 


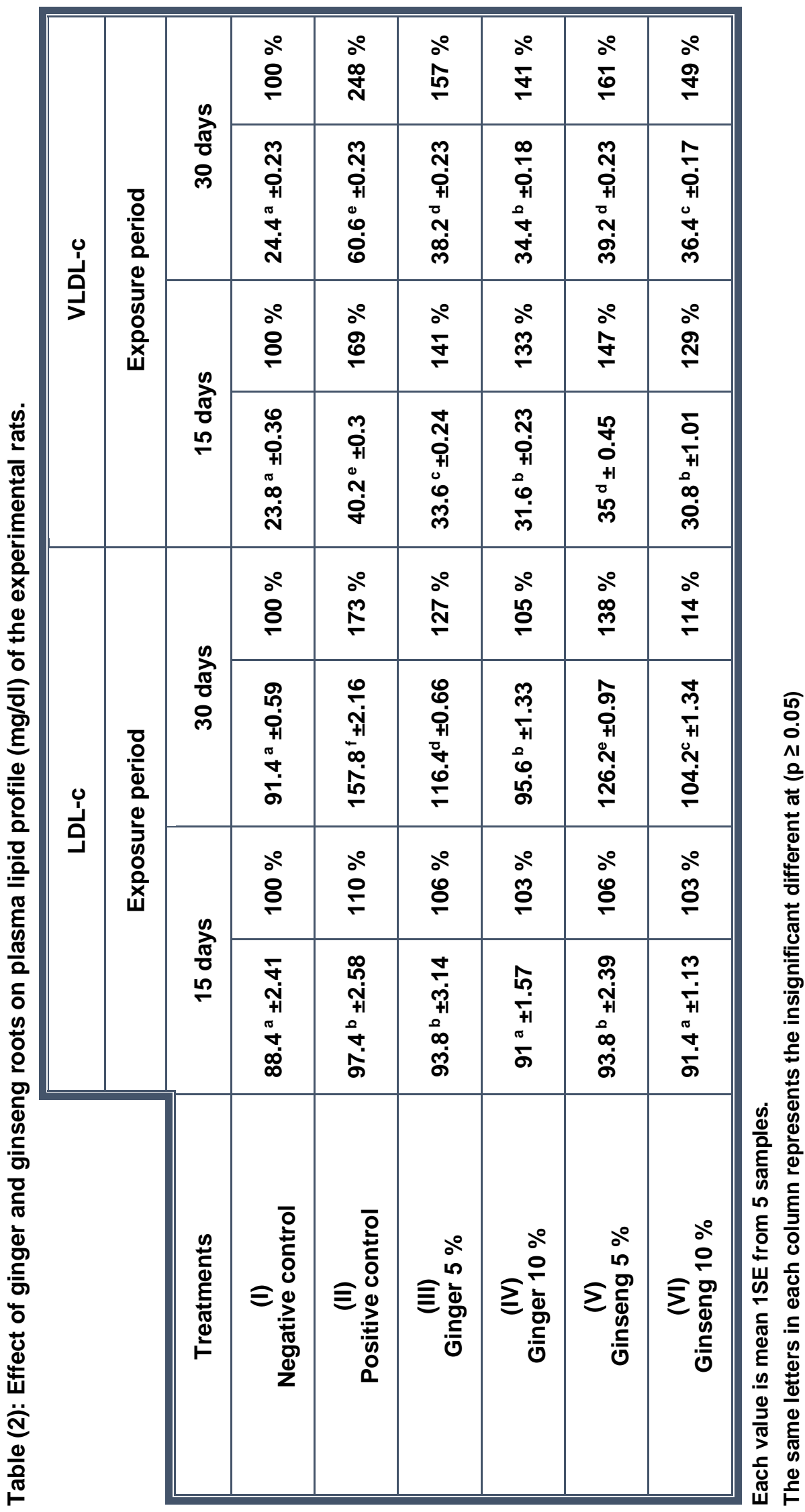




\begin{tabular}{|c|c|c|c|c|c|c|c|c|}
\hline \multirow{4}{*}{$\underset{\frac{1}{2}}{\stackrel{\partial}{2}}$} & \multirow{4}{*}{ 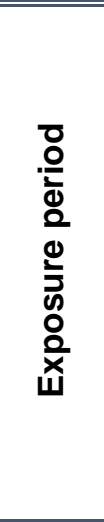 } & \multirow{2}{*}{$\begin{array}{l}\stackrel{0}{7} \\
\frac{\pi}{0} \\
\text { D }\end{array}$} & $\begin{array}{l}0 \\
8 \\
0\end{array}$ & $\begin{array}{l}\text { o̊ } \\
\text { ì } \\
\text { in }\end{array}$ & 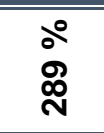 & $\begin{array}{l}\text { ○̊ } \\
\infty \\
\stackrel{2}{0}\end{array}$ & $\begin{array}{l}\circ \\
\infty \\
\infty \\
\text { m }\end{array}$ & 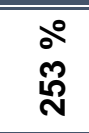 \\
\hline & & & مَ & ัั & 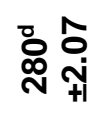 & 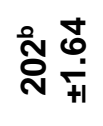 & 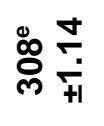 & 䖞 \\
\hline & & \multirow{2}{*}{ 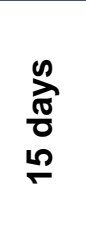 } & $\begin{array}{l}0 \\
8 \\
0\end{array}$ & $\begin{array}{l}\text { 尺े } \\
\text { స్ }\end{array}$ & $\begin{array}{l}\text { o̊ } \\
\text { in } \\
\text { N }\end{array}$ & $\begin{array}{l}0 \\
-1 \\
-1 \\
0\end{array}$ & $\stackrel{\circ}{\stackrel{0}{*}}$ & 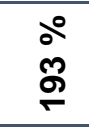 \\
\hline & & & 妿 $\frac{7}{+1}$ & 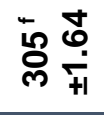 & Цొّ & 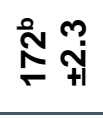 & స్ّ & 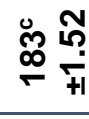 \\
\hline \multirow{2}{*}{$\frac{\stackrel{5}{\frac{1}{5}}}{\frac{5}{6}}$} & \multirow{2}{*}{ 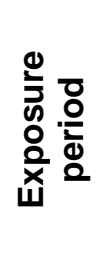 } & 이 & 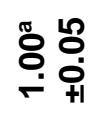 & 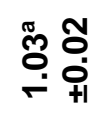 & 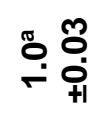 & 审 & 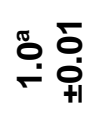 & 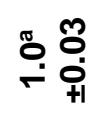 \\
\hline & & 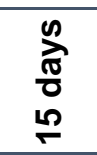 & 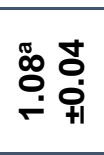 & 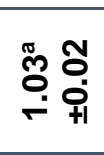 & 隽 & 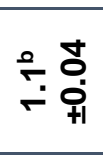 & 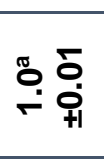 & 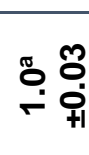 \\
\hline \multirow{4}{*}{$\underset{\frac{1}{2}}{\stackrel{一}{2}}$} & \multirow{4}{*}{ 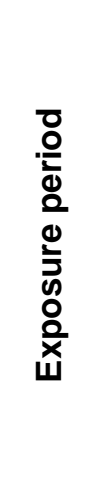 } & \multirow{2}{*}{ 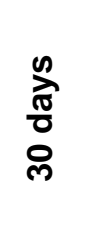 } & $\begin{array}{l}0 \\
8 \\
0\end{array}$ & $\begin{array}{l}\text { ১े } \\
\text { त् }\end{array}$ & $\begin{array}{l}\stackrel{0}{ } \\
\infty \\
\stackrel{\mathscr{N}}{N}\end{array}$ & $\begin{array}{l}0 \\
\stackrel{0}{0}\end{array}$ & 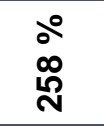 & $\begin{array}{l}\circ \\
\stackrel{9}{9} \\
\stackrel{9}{1}\end{array}$ \\
\hline & & & 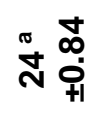 & $\stackrel{+}{\stackrel{*}{+}}$ & 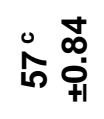 & 字 & 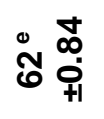 & チ \\
\hline & & \multirow{2}{*}{ 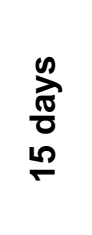 } & $\begin{array}{l}\circ \\
\text { o̊ }\end{array}$ & $\begin{array}{l}\stackrel{\circ}{ } \\
\text { Lึ } \\
\text { N }\end{array}$ & 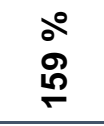 & $\begin{array}{l}\stackrel{0}{ } \\
\text { in }\end{array}$ & $\begin{array}{l}0 \\
\stackrel{N}{ }\end{array}$ & $\begin{array}{l}0 \\
\infty \\
0 \\
0 \\
-1\end{array}$ \\
\hline & & & $\stackrel{\infty}{N} \stackrel{\infty}{\substack{++1}}$ & 芯 & ن & స్లె & 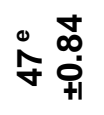 & 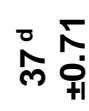 \\
\hline \multirow{4}{*}{$\underset{\frac{1}{2}}{\stackrel{5}{2}}$} & \multirow{4}{*}{ 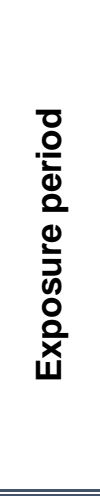 } & \multirow{2}{*}{ 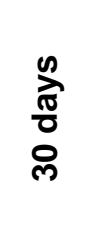 } & $\begin{array}{l}0 \\
8 \\
8\end{array}$ & $\begin{array}{l}\stackrel{0}{ } \\
\text { in } \\
\stackrel{\text { m }}{n}\end{array}$ & $\begin{array}{l}\stackrel{0}{ } \\
\stackrel{\text { N }}{N}\end{array}$ & $\begin{array}{l}\circ \\
10 \\
0 \\
\text { 엉 }\end{array}$ & $\begin{array}{l}\stackrel{0}{ } \\
\stackrel{+}{*} \\
\text { N }\end{array}$ & $\begin{array}{l}\stackrel{0}{ } \\
\text { హิ }\end{array}$ \\
\hline & & & ¿ & 萬 & 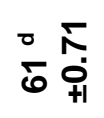 & 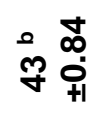 & $\begin{array}{l}0 \\
0 \\
0 \\
0 \\
0 \\
0\end{array}$ & $\begin{array}{l}+1 \\
0 \\
0 \\
0\end{array}$ \\
\hline & & \multirow{2}{*}{ 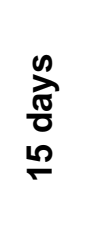 } & $\begin{array}{l}0 \\
8 \\
\stackrel{8}{9}\end{array}$ & $\begin{array}{l}0 \\
\text { ㅇ }\end{array}$ & $\begin{array}{l}\circ \\
\infty \\
0 \\
0 \\
\end{array}$ & $\begin{array}{l}0 \\
\text { d } \\
\text { a }\end{array}$ & $\begin{array}{l}\stackrel{0}{0} \\
\stackrel{9}{9}\end{array}$ & $\begin{array}{l}0 \\
0 \\
0\end{array}$ \\
\hline & & & 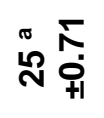 & $\stackrel{+1}{+}$ & ¿ & ํㅐㅇ $\underset{+1}{7}$ & 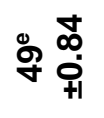 & $\begin{array}{l}0 \\
\dot{9}+1\end{array}$ \\
\hline & & 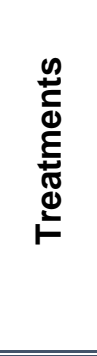 & 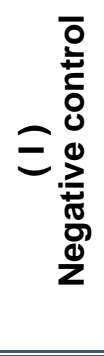 & 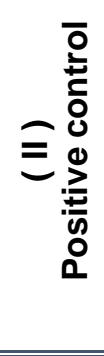 & 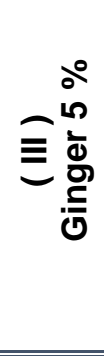 & 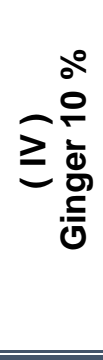 & 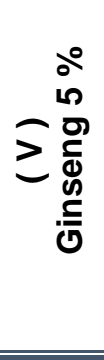 & 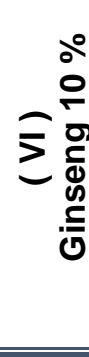 \\
\hline
\end{tabular}




\begin{tabular}{|c|c|c|c|c|c|c|c|c|}
\hline \multirow{4}{*}{ 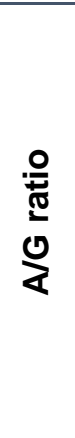 } & \multirow{4}{*}{ 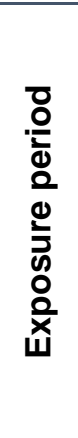 } & \multirow{2}{*}{ 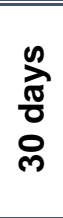 } & 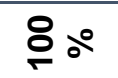 & 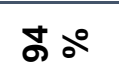 & §̊ ১゚ & $\underset{\text { S }}{\circ}$ & $\stackrel{\circ}{\circ}$ & స్తి \\
\hline & & & 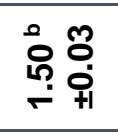 & 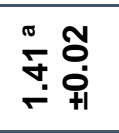 & 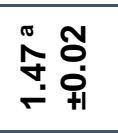 & 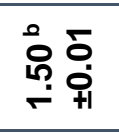 & 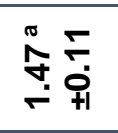 & 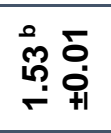 \\
\hline & & $\stackrel{n}{\geqslant}$ & 어 ১0 & 동 웅 & வீं 。ீ & ஸ゚ & กึ่ & તું \\
\hline & & 웅 & 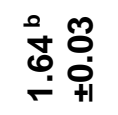 & 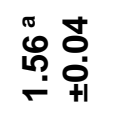 & 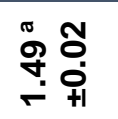 & 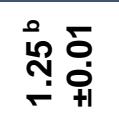 & 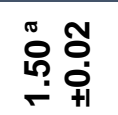 & 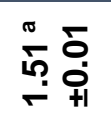 \\
\hline & & $\stackrel{n}{\text { డ }}$ & $\begin{array}{l}\circ \\
8 \\
0\end{array}$ & 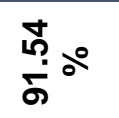 & $\begin{array}{l}\text { L̊ } \\
\text { ูㅇㅇ }\end{array}$ & 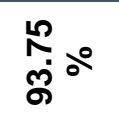 & 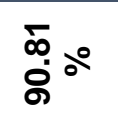 & $\begin{array}{l}\infty \\
\text { नै } \\
\text { ت̇ }\end{array}$ \\
\hline 흠 & 을 & ஓ & 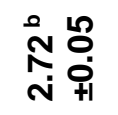 & $\begin{array}{l}\text { o } \\
\text { ơ } \\
\text { No }\end{array}$ & 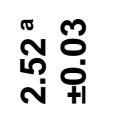 & 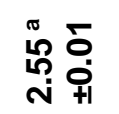 & $\begin{array}{l}\text { 先告 } \\
\text { No }\end{array}$ & 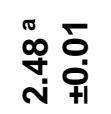 \\
\hline$\frac{\overline{\bar{J}}}{\frac{0}{0}}$ & $\begin{array}{l}\bar{y} \\
0 \\
\frac{0}{x} \\
\dot{x}\end{array}$ & 疍 & $\begin{array}{l}\circ \\
\stackrel{9}{0} \\
0\end{array}$ & 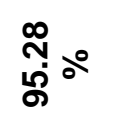 & $\stackrel{\infty}{\stackrel{1}{i}} \stackrel{0}{\circ}$ & 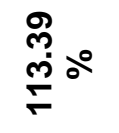 & $\begin{array}{l}0 \\
\stackrel{9}{0} \\
\stackrel{-}{1}\end{array}$ & مُ \\
\hline & & $\stackrel{10}{\rightarrow}$ & 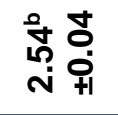 & 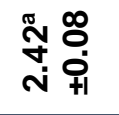 & 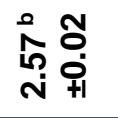 & 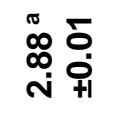 & 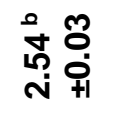 & 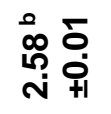 \\
\hline & & n & 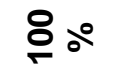 & $\notin \circ$ & ฮ่ & 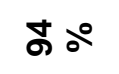 & ஃ ஃீ & g̊ \\
\hline 흥 & $\frac{0}{\frac{0}{0}}$ & $\begin{array}{l}\overline{0} \\
\text { ò }\end{array}$ & $\begin{array}{ll} & -1 \\
\infty & 0 \\
0 & 0 \\
\dot{+} & 0 \\
+ & +1\end{array}$ & 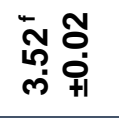 & 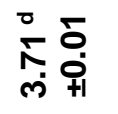 & 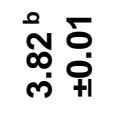 & 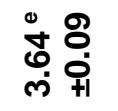 & 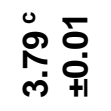 \\
\hline$\frac{0}{\bar{\alpha}}$ & $\begin{array}{l}\overline{7} \\
0 \\
0\end{array}$ & $\stackrel{n}{\circledR}$ & $\stackrel{\circ}{\circ}$ ㅇ & ㅇํㅇ & సี่ & นึ่ ๖ำ & कై & ભ̆ $_{\sigma}^{\infty}$ \\
\hline & & $\stackrel{0}{\circ}$ & $\begin{array}{ll}2 & -1 \\
0 & 0 \\
\ddots 1 & 0 \\
\dot{\gamma}+1\end{array}$ & 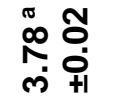 & 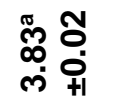 & 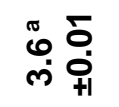 & $\begin{array}{ll} & -1 \\
\infty & 0 \\
\infty & 0 \\
& 0\end{array}$ & 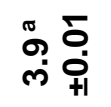 \\
\hline & & n & $\underset{-}{8}$ ১ & $\stackrel{\infty}{\infty}$ ○ & สั & హ。 & ৪ ১ & สั ๙゚ \\
\hline 흥 & 음 & $\begin{array}{l}\frac{\pi}{0} \\
\text { o }\end{array}$ & 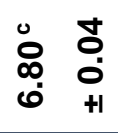 & 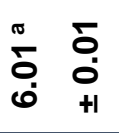 & 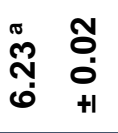 & 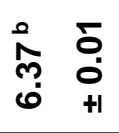 & 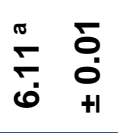 & 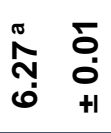 \\
\hline$\stackrel{a}{r}$ & $\begin{array}{l}\overline{\bar{\sigma}} \\
0 \\
0\end{array}$ & $\stackrel{n}{\lambda}$ & $\underset{-}{8}$ ○ & สู่ & นึ่ & क̊ㅇㅇ & $\begin{array}{l}0 \\
\text { sं }\end{array}$ & $\hat{\mathscr{o}}$ \\
\hline & ш & 它 & 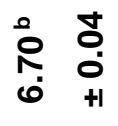 & \begin{tabular}{cc} 
O̊ & \multicolumn{0}{c}{} \\
\multirow{N}{*}{} & 0 \\
0 & +1
\end{tabular} & \begin{tabular}{ll}
$\tilde{0}$ & \multicolumn{1}{c}{} \\
o & 0 \\
0 & 0 \\
0 & +1
\end{tabular} & \begin{tabular}{cc}
0 & -1 \\
\multirow{0}{0}{} & 0 \\
0 & 0 \\
0 & +1
\end{tabular} & 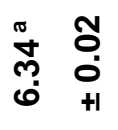 & 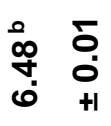 \\
\hline & & 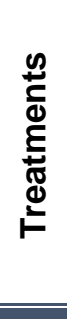 & 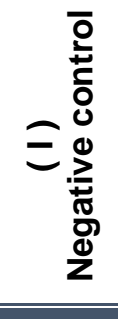 & 总 & 䓃点 & 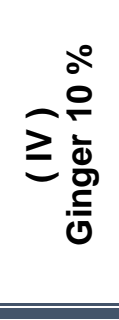 & 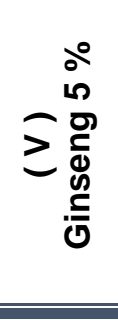 & 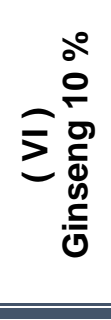 \\
\hline
\end{tabular}


The level of serum total protein significantly reduction ranged between $94.6-96.7 \%$ after 15 days and between $90-94 \%$ after 30 days comparing with normal control $100 \%$.

The globulin increased in most of tested groups. A/G ratio showed that ginger $10 \%$ and ginseng $10 \%$ recorded the higher ratio comparing with negative control. Venkateswarly et al., (1993). Found that there was a marked decrease in serum protein of hypercholesterolemic rats comparing with normal control animals.

\section{REFERENCE}

Ahmed, R.S., V. Seth and B. D. Banerjee (2000). Influence of dietary ginger (Zingiber officinales Rosc.) on antioxidant defense system in rat: Comparison with ascorbic acid. Indian J. Exp. Biol. 38(6): 604-606.

Al-Amin, Z. M., M. Thomson, K. K. AlQattan, R. Peltonen-Shalaby and M. Ali (2006). Anti-diabetic and hypolipidaemic properties of ginger (Zingiber officinale) in streptozotocininduced diabetic rats. $\mathrm{Br}$. J. Nutr.96(4):660-666.

Arif, B., J.M. Ashraf, A. J. Moinuddin, Z. Arif and K. Alam (2012). Structural and immunological characterization of amadori-rich human serum albumin: Role in diabetes mellitus. Arch Biochem Biophys.522:17-25

Bhandari, U., J.N. Sharma and R. Zafar (1998). The protective action of ethanolic ginger (Zingiber officinale) extract in cholesterol fed rabbits. J. Ethnopharmacol.;61(2):167-171.

Cannon, D.C., I. Olitzky and J.A. Inkpen (1974). Proteins. In: Clinical chemistry, principles and technics. 2-nd ed. RJ Henery. DC Cannon, JW Winkelman, editors, Harper and Row, New York, pp 407- 421.
El-Abd, M. M., M. N. Hassan, G. E. ElBaroty and A.E. Abd El-Mobdy (2007). Evaluation of conjugated linoleic acid (CLA) isomers as antiglycemic agents in albino rats. Egyptian J. Nutr.; 22(4): 261-288.

Fiedewaid, W.T., L.I. Levy and D.S. Fredrickson (1972). Estimation of concentration of low-density lipoprotein cholesterol in plasma without use of the preparative ultracentrifuge. Clin. Chem.; 18:499502.

Fossati, F. and L. Prencipe (1982). Plasma triglycerides determined colorimetrically with an enzyme that produces hydrogen peroxide. J.Clin.Chem, 28 (10): 2077 - 2080.

Fuhrman, B., M. Rosenblat, T. Hayek, R. Coleman and M. Aviram (2000). Ginger extract consumption reduces plasma cholesterol, inhibits LDL oxidation and attenuates development of atherosclerosis in atherosclerotic, apolipoprotein E-deficient mice. J Nutr.130(5):1124-1131.

Gopalan, C., S.B.V. Rama, S.C. Balasubramanian, N.B.S. Rao, Y.G. Deosthale and K.C. Pant (2004). Nutritive value of indian foods. Hyderabad, National Institute of Nutrition Indian Council of Medical Research

Islam, M.S. and H. Choi (2008). Comparative effects of dietary ginger (Zingiber officinale) and garlic (Allium sativum) investigated in a type 2 diabetes model of rats. J. Med. Food.11(1):152-159

Kim, T.S., Y.J. Kim, S.A. Jang, K.H. Yang, N.K. Seung and E.H. Sohn (2012). Protective effects of red ginseng against radiation-induced hepatotoxicity in mice. Proceedings of the Spring International Ginseng Conference; April; Jeju, Korea. The Korean Society of Ginseng; p. p. 100. 
Landue, S. and B.S. Everitt (2004). A Handbook of statistical Analyses using SPSS. -London: Chapman \&Hall/CRC press LLC, 2004. 337p.

Lee, S.T., K. Chu, J.M. Kim, H.J. Park and M. Kim (2006). Cognitive improvement by ginseng in Alzheimer' disease. Proceedings of the 9th International Ginseng Symposium; 2006. Geumsan, Chungnam, Korea, Korean Society of Ginseng.

Lopez, M.F., S. Stone, S. Ellis and J.A. Collwell (1977). Cholesterol determination in high-density lipoproteins separated by three different methods. Clin.Chem. 23(5):882 - 886.

Luo, D. and B. Fange (2008). Structural identification of ginseng polysaccharides and testing of their antioxidant activities. Carbohyr. Polym. 72:376-381

Matsuda, A., Z. Wang, S. Takahashi, T. Tokuda, N. Miura and J. Hasegawa (2009). Upregulation of mRNA of retinoid binding protein and fatty acid binding protein by cholesterol enriched-diet and effect of ginger on lipid metabolism. Life Sci.84(2526):903-907.

Moss, D.W., A.R. Henderson and J.F. Kachmar (1987). Enzymes in: Tietz $\mathrm{NW}$, ed. Fundamentals of clinical chemistry. 3 rd ed. Philadelphia: WB Saunders; 346-421.

Murray, R.K., D.K. Grenner and V.W. Rodwell (2006). Harpers illustrated biochemistry 27 th ed. MC-Graw. Hill Education Casia PP 70-101.

Nadeem, A.A., Moinuddin, K. Alam and A. Ali (2009). Preferential recognition of Amadori-rich lysine residues by serum antibodies in diabetes mellitus: Role of protein process in the disease process. Hum. Immunol.70:417-424.
Nicoll, R. and M.Y. Henein (2009). Ginger (Zingiber officinale Roscoe): A hot Remedy for Cardiovascular Disease? Int. J. Cardiol, 131: 408-409.

Niu, T., Y.J. Liu, L.X. Tian, K.S. Mui, H. Yang, C.X. Ye and Y. Zhu (2008). Effects of dietary phospholipids level in cobia (rachycentron canadum) larvae: growth, survival, plasma lipids and enzymes of lipid metabolism. Fish physical. Biochem, 34(1): 9-17.

Sang, M.L., B. Bong-s, W.P. Hee, G.A. Nam, Cu. Byung and Y.S. Yong (2015). Characterization of Korean Red Ginseng (Panax ginseng Meyer): History, preparation method, and chemical composition. JGR J. Ginseng Res. 39: 384-391.

Schultze, H.E. and J.F. Heremans (1966). Molecular biology of human protein. Elsevier publishing company, Amsterdam.1: section 3, chap3.

Venkateswarly, V., C. K. Kokate, D. Rambhan and C. Veereshan (1993). Antidiabetic activity of roots of Salacia macrosperma. Planta Med. 59:339-393

Young, D.S. (1990). Effects of Drugs on Clinical Laboratory Tests, AACC Press, Washington DC, Third edition. 3:6 -12.

Zhang, X., L. Yu, H. Bi, X. Li, W. Ni, H. Han, N. Li, B. Wang, Y. Zhou and G. Tal (2009). Total fractionation and characterization of the water soluble polysaccharides isolated from Panax ginseng C,A. meyer. Carbohydr. Polym. 77: 544-552. 
تأثير ريزومات الزنجبيل والجنسنج على وظائف الكبد في الفئران المصابة بارتفاع الدهون

محمد عبد السلام حبي، أحمد محمد فريد، هلى السيد أحمد فريد، شعبان حامد محمد حبيثة الزية

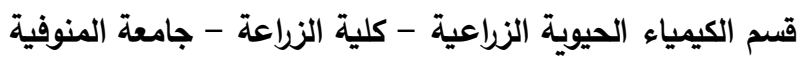

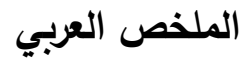

لقد تم استخدام ريزومات الزنجبيل والجنسنج تركيز ه \& \& ـ ـ 1 في غذاء الفئران المصابة بارتفاع

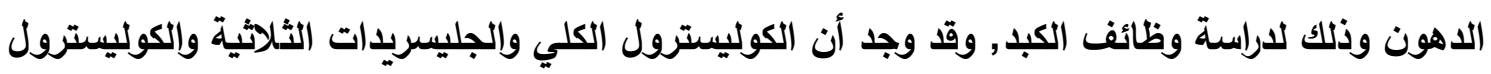

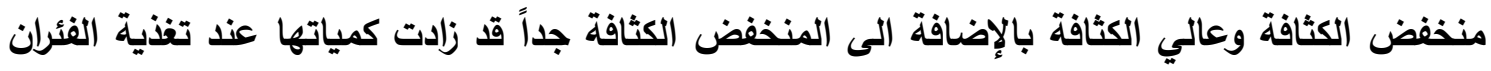

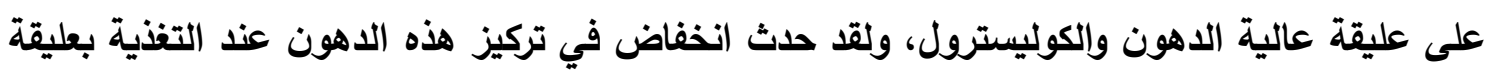
تحتوي على الزنجبيل أو الجنسنج.

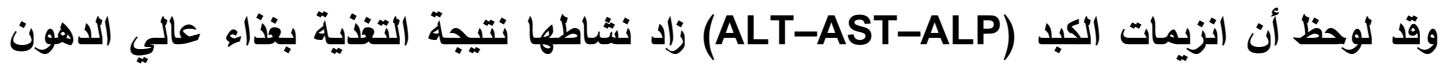

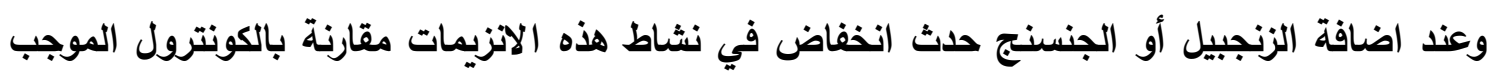

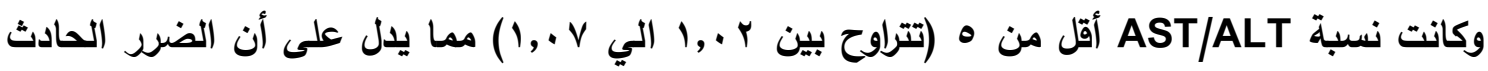

$$
\text { كان في الكبد وليس القلب. }
$$

ظهر أيضاً انخفاض في كمية البروتين الكلي والألبومين نتيجة التغذية بغذاء عالي الدهون وعند التهن اضافة الزنجبيل أو الجنسنج الى العليقة زادت كمية البروتين مقارنة بالكونترول الموجب. 